\title{
Variaciones previsibles del ingreso y su efecto sobre el consumo. \\ Un análisis para la economía colombiana*
}

\section{Predictable variations of income and its effect on consumption.}

An analysis for Colombian economy

Nicolás Ronderos Pulido**

Recibido: 20 de Noviembre de 2019

Revisado: 28 de Enero de 2019

Aprobado: 5 febrero de 2019

* Este artículo hace parte del proyecto ingreso y consumo en la economía colombiana. Cómo citar este artículo: Ronderos, N. (2019). Variaciones previsibles del ingreso y su efecto sobre el consumo. Un análisis para la economía colombiana. Revista CIFE: Lecturas de Economía Social, 20(33), 17-37. DOI: https://doi.org/10.15331/22484914.4874

** Economista y magíster en economía de la Pontificia Universidad Javeriana. Docente investigador en la Universidad Santo Tomás. Correo electrónico: nicolasronderos@usantotomas.edu.co. 


\section{Resumen}

En este trabajo se realiza una revisión de las teorías que explican los determinantes del consumo de los hogares. Con base en variaciones estacionales del ingreso de la economía colombiana se realiza una estimación del ingreso previsible. Utilizando un modelo $\operatorname{VAR}(\mathrm{p})$ se encuentra evidencia a favor de la relación entre ingreso previsible hacia el consumo de los hogares, se apoya la llamada hipótesis de exceso de sensibilidad del consumo (Flavin, 1981). Se concluye que para la economía colombiana los consumidores no incorporan toda la información disponible para establecer su senda de gastos. Por lo cual, las variaciones previsibles en el ingreso disponible, causadas por cambios en el nivel de impuestos o subsidios, generan variaciones adicionales en las compras agregadas de los hogares.

Palabras clave: consumo, ingreso previsible, series de tiempo, contabilidad de innovaciones.

Clasificación JEL: E21, C32

\section{Abstract}

In this paper a review of the theories that explain the determinants of household consumption is performed. Based on seasonal variations in income of the Colombian economy, an estimate of predictable income is made. Using a $\operatorname{VAR}(\mathrm{p})$ model, evidence is found in favor of the relationship between predictable income to household consumption, the hypothesis of excess sensitivity of consumption is supported (Flavin, 1981). It is concluded that for the Colombian economy, consumers do not incorporate all the available information to establish their consumption path. Therefore predictable variations in the available income, caused by changes in the level of taxes or subsidies, generate additional variation in aggregate purchases of the household.

Keywords: Consumption, predictable income, time series, innovation accounting.

Classification JEL: E21, C32 


\section{Introducción}

En cualquier economía del mundo el consumo de los hogares representa una gran proporción del GNB, por ello los determinantes del consumo de los hogares han sido ampliamente investigados en la literatura. El estudio del consumo inició con el planteamiento de Keynes (1936), el cual establece que el nivel de ingreso actual es el principal determinante del consumo y ahorro de los hogares. La evidencia empírica actual demuestra que existen periodos en donde el consumo y el ahorro presentan grandes desviaciones con respecto a lo que predice el nivel de ingreso actual (Rebeco, 2007). A causa de esto, el planteamiento de Keynes es reformulado en varias ocasiones. Friedman (1957) propone la teoría del ingreso permanente, en la cual un individuo determina su consumo óptimo distribuyéndolo uniformemente en el tiempo, bajo la premisa de que un individuo establece su senda futura de ingresos y gastos sin incertidumbre. Por esta razón los cambios en el ingreso permanente tendrán la suficiente influencia en la senda de ingreso como para afectar el nivel de consumo promedio. Esta teoría es un caso particular del modelo de consumo intertemporal de Irving Fisher (Mankiw, 2014; Rebeco, 2007; Romer, 1996), en el cual un individuo determina su senda óptima de consumo de acuerdo con sus preferencias de consumo intertemporal y la tasa de interés real de sus depósitos y créditos. La tasa real se utiliza para calcular el valor presente de los ingresos futuros. Estas teorías explican las razones por la cuales los datos macroeconómicos del consumo fluctúan suavemente en el tiempo y usualmente con menor variación que el nivel agregado de ingreso. Además, establecen que cambios en el ingreso disponible causados por impuestos transitorios tendrán una menor influencia que los impuestos permanentes.

Por otro lado, desde la hipótesis de paseo aleatorio propuesta por Hall (1978), se establece la importancia de determinar si variaciones previsibles del ingreso generan cambios en el consumo, a causa de que dicha hipótesis plantea que los cambios en la senda de consumo de un individuo se encuentran determinados por choques estocásticos e independientes de los usuales determinantes Bdel consumo. Así bien, tanto la teoría de ingreso permanente como la hipótesis de paseo aleatorio predicen que no existe relación entre el ingreso previsible y el consumo. La teoría de ingreso permanente establece que un individuo usa su ahorro para suavizar su senda de consumo y optimiza de acuerdo con su senda de ingreso conocida con total certidumbre, por ello la senda de consumo incorpora la información del ingreso futuro, de esta manera los cambios en el ingreso previsible no deberían generar cambios en el consumo.

Existe evidencia microeconométrica que apoya la existencia de la relación causal entre ingreso previsible hacia el consumo (Agarwal y Souleles, 2007; Attanasio y Weber, 2013; Johnson, Parker, 1999; Parker y Souleles, 2006; Parker, Souleles, Johnson y McClelland, 2013; Shapiro y Slemrod, 2003; Shea, 1995). Sin embargo, a nivel agregado la evidencia 
es contradictoria. En algunos trabajos se apoya la evidencia a favor de la relación causal (Campbell y Mankiw, 1989; Carroll, 1997; Paxson, 1992), mientras que en otros la evidencia de esta relación es inexistente (Browning y Colado, 2001; Hsieh 2003; Paxson, 1993). A diferencia de las teorías de ingreso permanente, paseo aleatorio y el modelo de Fisher existen varios motivos que explican la existencia de dicha relación: i) ahorro precautelativo, ii) restricciones de liquidez, iii) alejamiento de la optimización de los consumidores y iv) formación de hábitos de consumo.

Este trabajo se enfoca en establecer una relación entre ingreso previsible y consumo de los hogares a nivel agregado para el caso de la economía colombiana. Contiene cuatro secciones incluyendo esta introducción.

En la segunda sección se expone un modelo teórico y una revisión de literatura que explica la existencia de dicha relación causal.

En la tercera sección se presentan las estimaciones utilizando como medida de ingreso previsible ciclos estacionales fijos en el ingreso. En la última sección se muestran las conclusiones y recomendaciones del estudio.

\section{Marco teórico}

A continuación, se plantea un modelo que explica la relación entre ingreso previsible y consumo. Suponga que un individuo no posee activos en el momento $t$, su horizonte de vida es infinito y decide mantener un consumo constante en el tiempo. Además, se asume que en el momento cero el individuo tiene un ingreso constante e igual a $Y_{\mathrm{y}}$ con probabilidad $p_{r}$ su ingreso se mantendrá en $Y$ durante su vida. En el momento uno con una probabilidad $p_{j}$, el ingreso podrá cambiar a un nivel $Y_{j}$ para $j=1, \ldots, n-1$ durante su vida. Por lo que existen n posibles resultados de ingreso ${ }^{1}$.

La restricción de consumo intertemporal del individuo se encuentra dada por la siguiente expresión (Romer, 1996; Rebeco, 2007; Mankiw, 2014).

$$
\sum_{i=0}^{\infty} \frac{C_{t+i}}{(1+r)^{i}}=(1+r) A_{t}+\sum_{i=0}^{\infty} \frac{Y_{t+i}}{(1+r)^{i}}
$$

1 Las probabilidades de ocurrencia se encuentran determinadas por la distribución de probabilidad del ingreso. Como es bien sabido esta presenta asimetría positiva. Por simplicidad se plantea una función de distribución del ingreso discreta. 
Así, el valor presente del consumo es igual al valor presente del ingreso. Bajo la situación de ingreso constante e igual a $Y$ el valor presente del ingreso se encuentra dado por:

$$
V p(\bar{Y})=\sum_{i=0}^{\infty} \frac{\bar{Y}}{(1+r)^{i}}
$$

Teniendo en cuenta que $0 \leq \frac{1}{(1+r)^{i}} \leq 1$ la anterior expresión es equivalente a la siguiente ecuación.

$$
V p(\bar{Y})=\frac{\bar{Y}}{1-\frac{1}{1+r}}=\frac{\bar{Y}(1+r)}{r}
$$

Si en el momento uno el ingreso es igual a $\Upsilon_{j}$, el valor presente del ingreso es igual a la siguiente expresión.

$$
V p\left(Y_{j}\right)=\bar{Y}+\sum_{i=1}^{\infty} \frac{Y_{j}}{(1+r)^{i}}
$$

Donde $\sum_{i=1}^{\infty} \frac{1}{(1+r)^{i}}=\frac{1+r}{r}-1=\frac{1}{r}$. Se tiene que el valor presente del ingreso está dado por la siguiente igualdad para $j=1, \ldots, n-1$.

$$
V p\left(Y_{j}\right)=\bar{Y}+\frac{Y_{j}}{r}
$$

De acuerdo con la restricción de consumo intertemporal, el valor presente del consumo es igual al valor presente del ingreso, en este contexto el valor presente del consumo es igual al valor esperado del valor presente del ingreso.

$$
\frac{C(1+r)}{r}=\frac{p_{\bar{Y}} \bar{Y}(1+r)}{r}+\sum_{j=1}^{n-1} p_{j}\left(\bar{Y}+\frac{Y_{j}}{r}\right)
$$

Lo cual puede expresarse como:

$$
C=p_{\bar{Y}} \bar{Y}+\frac{1}{1+r} \sum_{j=1}^{n-1} p_{j}\left(\bar{Y} r+Y_{j}\right)
$$


La propensión marginal por consumir (PMC) es una medida de asociación entre consumo e ingreso. Con la expresión del consumo puede obtenerse la PMC de este individuo en el momento $t=1$, de la forma $\frac{C_{1}-C_{0}}{Y^{*}-\bar{Y}}$. Donde $Y^{*}=\frac{\sum_{j=1}^{n-1} p_{j} Y_{j}}{\sum_{j=1}^{n-1} p_{j}}$ es el valor esperado del ingreso excluyendo la posibilidad del ingreso constante, ya que bajo esta situación la PMC no se encuentra definida. Considerando que $C_{0}=r$.

$$
C=p_{\bar{Y}} \bar{Y}+\frac{1}{1+r} \sum_{j=1}^{n-1} p_{j}\left(\bar{Y} r+Y_{j}\right)
$$

Además, dado que $\sum_{j=1}^{n} p_{j}=1$.

$$
C_{1}-C_{0}=\frac{1}{1+r} \sum_{j=1}^{n-1} p_{j}\left(Y_{j}-\bar{Y}\right)
$$

Por lo cual, después de un poco de álgebra, la PMC se encuentra dada por la siguiente ecuación.

$$
\frac{C_{1}-C_{0}}{Y^{*}-\bar{Y}}=\frac{1}{1+r} \sum_{j=1}^{n-1} p_{j}=\frac{1-p_{\bar{Y}}}{1+r}
$$

Cuanto más probable sea que el ingreso cambie i.e $p_{r} \downarrow$ el consumo reaccionará en una mayor proporción a los cambios del ingreso. En el caso de que $p_{r}=0$ y la PMC sea igual a $\frac{1}{1+r}$, , es decir cuando el ingreso cambia con total certeza el consumo y el ingreso mantienen una correlación cercana a uno. En caso de que $r=0$ su correlación es igual a uno, dado que el valor presente del ingreso y el consumo es igual al valor futuro del ingreso y el consumo. En esta situación cambios previsibles en el ingreso tendrán influencia sobre el consumo, esto puede explicarse a causa de: i) ahorro precautelativo (Leland, 1968; Skinner, 1988) restricciones de liquidez (Deaton 1989; Zeldes, 1989) la inexistencia de expectativas racionales (Canzoneri, Cumby y Diba, 2007; Laibson, 1997). A continuación, se exponen estos tres puntos.

Si un hogar enfrenta incertidumbre con respecto a su senda futura de ingreso, este deberá prevenirla incrementando su ahorro como plan de contingencia. Por lo cual, cuanto mayor sea la incertidumbre ( $p_{r} \downarrow$ y las probabilidades sean más dispersas), mayor será el ahorro actual y por ende mayor será el consumo futuro. Las probabilidades del cambio en el ingreso pueden ser calculadas con su distribución de probabilidad. Ahora bien, si la incertidumbre sobre el ingreso aumenta $p_{r} \downarrow$, esto puede verse reflejado en una de dos 
situaciones: i) un incremento del ingreso a un nivel $\Upsilon_{j}>\Upsilon_{\mathrm{o}}$; ii) una disminución a un nivel $\Upsilon_{j}<\Upsilon$. Dado que la renta se encuentra concentrada en niveles bajos, es más probable que se observe un incremento en el ingreso, a causa de una alta PMC el incremento en el ingreso genera un aumento en el consumo futuro, así a mayor incertidumbre más consumo futuro. Y por tanto cambios previsibles en el ingreso ocasionan variaciones en el consumo.

Si un individuo se enfrenta a restricciones de liquidez relevantes, su consumo se encuentra limitado por su ingreso. Por lo cual las variaciones predecibles del ingreso afectan su consumo. Por ejemplo, en caso de que $p_{r}=0$, el ingreso disminuya y el individuo esté sujeto a una restricción de liquidez relevante, la disminución en el ingreso previsible genera a su vez una caída en su consumo $\left(P M C=\frac{1}{1+r}\right)$. El consumo podría permanecer en su nivel anterior si el individuo se endeudara.

Otro motivo por el cual el ingreso previsible se encuentra relacionado con el consumo, es que algunos individuos pueden no tener expectativas racionales. De esta manera la senda de consumo se plantea sin tener en consideración toda la información disponible. De esta forma las variaciones previsibles en el ingreso son vistas como nueva información para replantear la senda de consumo.

Por otro lado, en el caso de que el ingreso permanezca constante con total certeza i.e $p_{r}=1$ el ingreso no tendrá influencia alguna sobre el consumo. De tal forma que el ingreso previsible no tiene influencia sobre el consumo, ya que si el ingreso no varía no puede causar ningún cambio en el consumo. Esta situación es similar a la planteada en la hipótesis de paseo aleatorio (Hall, 1978).

\section{Estimación}

En este trabajo se estima un modelo $\operatorname{VAR}(\mathrm{p})$ con las siguientes dos variables endógenas: i) las ventas del comercio al por menor mensuales, como medida de consumo de los hogares y; ii) una estimación de ingreso previsible utilizando el Indicador de Seguimiento a la Economía (ISE) publicado por el DANE². Las dos cubren el periodo enero del 2000 hasta diciembre del 2017. El VAR(p) se calcula utilizando estas medidas debido a su frecuencia mensual. La agregación de series temporales puede generar relaciones de causalidad espurias y problemas para realizar inferencia estadística a causa de componentes VMA no invertibles (McCrorie y Chambers, 2006).

2 El ISE se construye con base en 111 indicadores coincidentes de las 9 ramas de actividad económica que componen el PIB. Su cálculo se realiza mediante un promedio ponderado de estos 111 indicadores, y sus ponderaciones se establecen de acuerdo con la participación del sector en el valor de la producción interna de la economía. 


\subsection{Ingreso previsible}

La estimación del ingreso previsible está basada en variaciones determinísticas del ingreso causadas por: bonificaciones a los trabajadores, gastos en matrículas escolares, la agenda tributaria, contratos a término fijo, efectos calendario y costumbres sociales. En la figura 1 se presenta la estimación del periodograma, basada en Priestley (1981), sobre la primera diferencia del $\mathrm{ISE}^{3}$ junto con el ISE en diferencias. Se puede observar que predominan las frecuencias fijas estacionales, usuales de observar en una serie de tiempo con periodicidad mensual.

El ingreso predictible $\Delta I S E^{p}$ se construye utilizando los valores ajustados de la siguiente regresión.

$$
\Delta I S E=\sum_{k=1}^{N_{o}} \alpha_{k} \operatorname{Cos}\left(w_{k} t\right)+\beta_{k} \operatorname{Sen}\left(w_{k} t\right)
$$

Donde $\mathcal{N}_{o}$ es el número de ondas sinusoidales utilizadas en la regresión. El término estocástico podría interpretarse erróneamente como la parte del ingreso no previsible, ya que este se encuentra autocorrelacionado. Flavin (1981) define la relación entre ingreso previsible y consumo como exceso de sensibilidad del consumo, y de forma similar define la relación entre ingreso imprevisible y consumo como exceso de suavidad del consumo. Cuanto mayor sea $\mathcal{N}_{o} \Delta I S E^{p}$ será cercano a $\triangle I S E$. El término $\mathcal{N}_{o}$ se selecciona con base en la significancia estadística conjunta de $\sqrt{\widehat{\alpha_{k}^{2}}+\widehat{\beta_{k}^{2}}}$, la amplitud de la onda. En la figura 2 se muestra la estimación de $\triangle I S E^{p}$ con base en distintos $\mathcal{N}_{0}$. Se puede observar que existe una alta relación entre el ingreso previsible y el ingreso, con una correlación cercana a 0.91 .

Cada una de las series de ingreso predictible estimadas se calcula como la suma de ondas sinusoidales, por lo que pueden ser pronosticadas sin error. En la figura 3 se muestran las ondas individuales que componen $\triangle I S E^{p}$ al $5 \%$ de significancia. Por definición la estimación del ingreso previsible es estacionaria. Las ondas de doce y seis meses por ciclo son las que presentan la mayor amplitud en términos numéricos. 
Figura 1. Periodograma sobre la primera diferencia del ISE
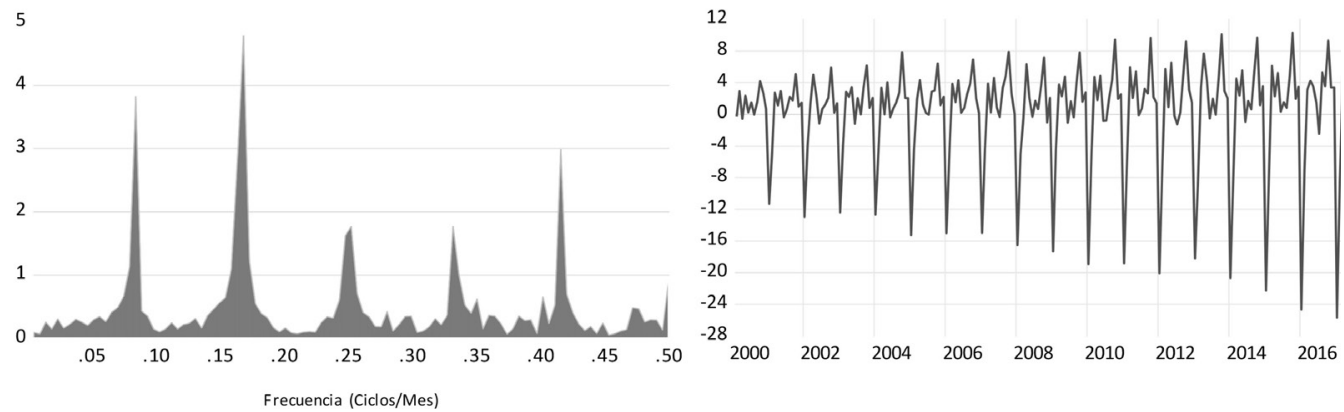

Fuente. DANE

Figura 2. Estimación del ingreso previsible

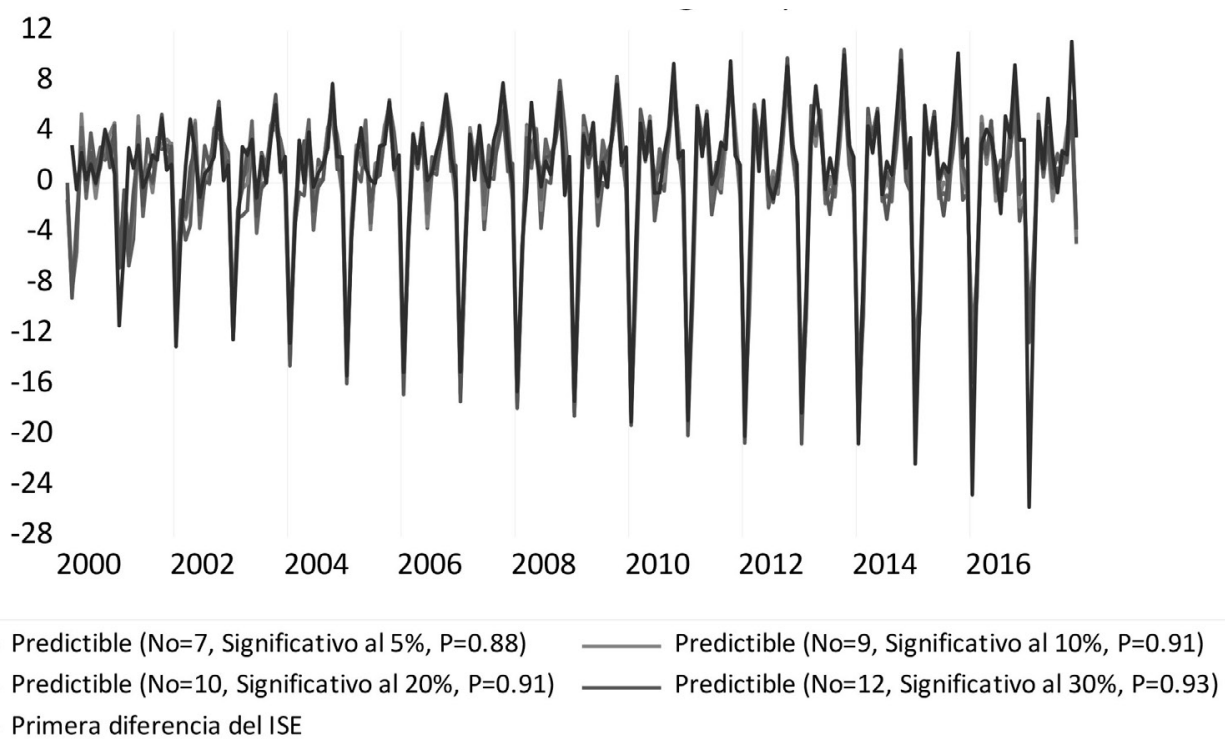

Fuente. Elaboración propia 
Figura 3. Ondas sinusoidales individuales
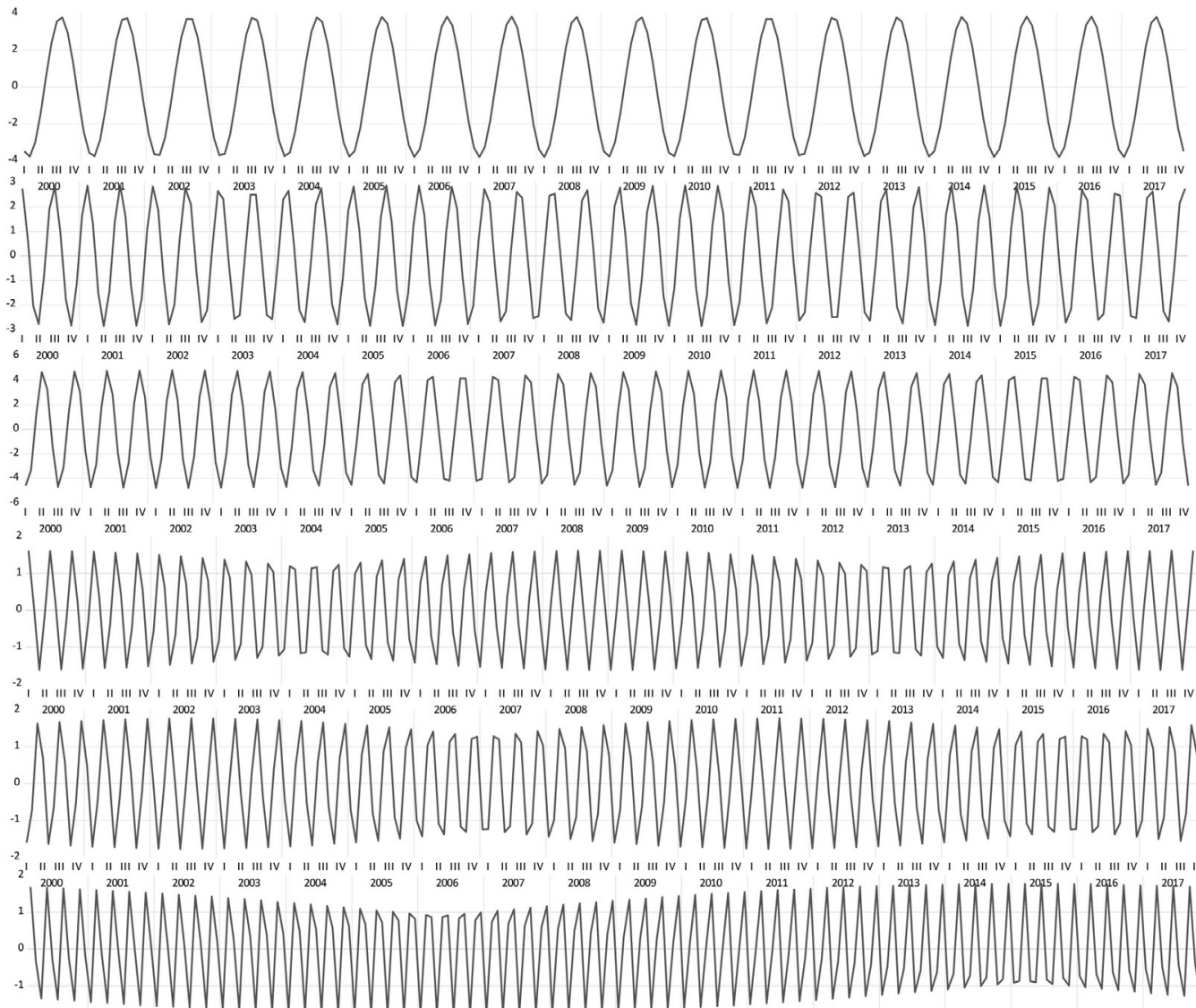

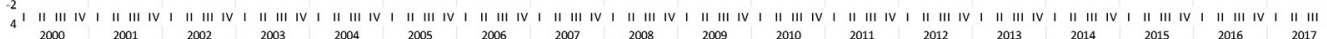

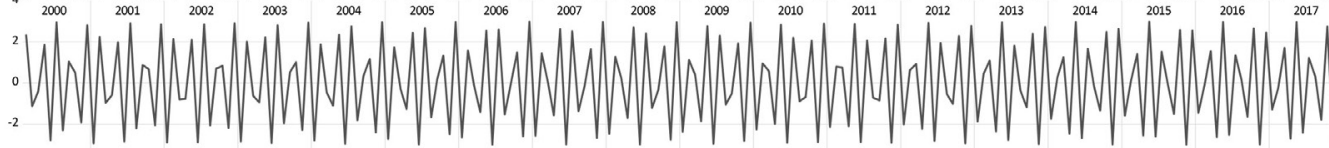

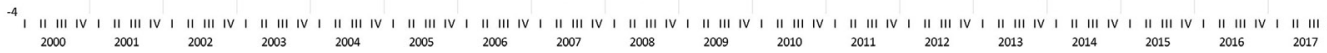

Fuente. Elaboración propia

\subsection{Vector autorregresivo}

Utilizando la estimación del ingreso previsible y la primera diferencia de las ventas minoristas ${ }^{4}$ se estima un modelo $\operatorname{VAR}(\mathrm{p})$ con variables dicótomas estacionales, con el objetivo de controlar por efectos estacionales que expliquen al ingreso previsible y a su vez se

4 Mediante las pruebas de raíz unitaria de Dickey-Fuller aumentada (ADF), Phillips-Perron (PP), Dickey-Fuller GLS, y Kwiatkowski-Phillip-Schmidt-Shin (KPSS) se determinó que la serie de tiempo de las ventas minoristas es integrada de orden uno. 
encuentren relacionados con el consumo. El orden p se fijó en 15 de acuerdo con la no autocorrelación conjunta del error. Es importante mencionar que el consumo agregado también se encuentra determinado por la tasa de interés de colocación y de captación, los impuestos gravados al consumidor, los subsidios, el nivel de confianza de los hogares con respecto a sus ingresos y gastos futuros, su capacidad de endeudamiento, la estructura demográfica del país y su sistema pensional. Estos factores se encuentran incluidos en los rezagos de las ventas minoristas en el VAR(p). En el anexo 1 se muestra la estimación del $\operatorname{VAR}(15)$ usando el ingreso previsible al $20 \%$ de significancia ${ }^{5}$.

Ahora bien, la estimación de la matriz de varianza covarianza de los errores es ortogonal, por lo cual una descomposición estructural es innecesaria y no afecta los resultados ${ }^{6}$ (Lütkepohl, 2005). En la tabla 1 se presenta la estimación de la matriz de varianza covarianza y de correlación de los errores. La varianza de los errores de la ecuación de $\Delta I S E^{p}$ es cercana a cero debido a su naturaleza determinista.

Tabla 1. Matriz de varianza covarianza de los errores del VAR

\begin{tabular}{cccc} 
(Correlación) [Covarianza] & $e t, \Delta I S E^{p}$ & \multicolumn{2}{c}{ et, $\Delta$ consumo } \\
\hline$e t, \Delta I S E^{p}$ & $(1.00)$ & - & - \\
$e t, \Delta$ consumo & $(-0.0029]$ & $(1.00)$ & {$[-0.0117]$}
\end{tabular}

Fuente. Elaboración propia

\subsection{Contabilidad de innovaciones}

Usando la estimación del $\operatorname{VAR}(15)$ se realizó un análisis de: i) descomposición de varianza del error de predicción; ii) descomposición histórica de las variables endógenas y; iii) un análisis de impulso y respuesta ${ }^{7}$. Las descomposiciones se realizan teniendo en cuenta que un $\operatorname{VAR}(\mathrm{p})$ puede expresarse como un $\operatorname{VMA}(\infty)$. Un $\operatorname{VAR}(\mathrm{p})$ con $m$ variables endógenas esta descrito de la siguiente forma.

$$
X_{t}=A_{0}+A_{t} X_{t-1}+\ldots+A_{p} X_{t-p}+e_{t}
$$

Y puede expresarse como un $\operatorname{VMA}(\infty)$ si su solución es convergente en términos del pasado y del presente de $e_{t}$. El VMA( $\left.\infty\right)$ está dado por la siguiente ecuación.

$$
X_{t}=e_{t}+B_{1} e_{t-1}+B_{2} e_{t-2}+\ldots
$$

5 Se ensayó modelos VAR usando el ingreso predictible calculado a otros niveles de significancia, desde el $5 \%$ hasta el $30 \%$. Los resultados no son sensibles a la elección de este parámetro.

6 Para el ejercicio de contabilidad de innovaciones de la sección 3.3 se estimaron modelos SVAR(p) y sus resultados se contrastaron con el VAR(p) sin restricciones. Los dos resultados en términos estadísticos no son diferentes.

7 Adicionalmente se realizaron pruebas de causalidad en el sentido de Granger. La hipótesis de no causalidad del ingreso previsible al consumo es rechazada a cualquier nivel de significancia. 
Este VMA $(\infty)$ a su vez puede calcularse en términos de errores estructurales ortogonales, tal que:

$$
X t=\Psi_{0} \varepsilon_{t}+\Psi_{1} \varepsilon_{t-1}+\ldots
$$

Donde $\Psi_{0}=P, \Psi B_{i}=B_{i} \mathrm{P}$ para $i=1, \ldots, \infty, \varepsilon_{t}=P^{-1} e_{t}$ y $P$ proviene de alguna descomposición estructural del término de error que garantice su ortogonalidad. Puede ser de un $\operatorname{SVAR}(\mathrm{p})$ o usualmente es tomada de la descomposición de Choleski, tal que $P P^{\prime}=\sum e$. En este caso $\sum e$ es ortogonal, por lo que la descomposición estructural es irrelevante. Puede demostrarse que el error de predicción está determinado por:

$$
\widehat{\varepsilon_{t+n}}=X_{t+n}-\widehat{X_{t+n}}=\sum_{j=0}^{n-1} \Psi_{j} \varepsilon_{t+n-j}
$$

$\mathrm{Al}$ pronosticar se asume que los errores son iguales a su valor esperado. Por lo que el error de pronóstico se determina por el valor de estos errores futuros desconocidos. De esta manera la varianza del error de pronóstico está dada por:

$$
\Sigma_{t+n}=E\left(\widehat{\varepsilon_{t+n}} \widehat{\widehat{t+n}^{\prime}}\right)=\Psi_{0} \Psi_{0}^{\prime}+\Psi_{1} \Psi_{1}^{\prime}+\cdots+\Psi_{n-1} \Psi_{n-1}^{\prime}
$$

A causa de que los errores estructurales son no autocorrelacionados y ortogonales i.e. $E\left(\varepsilon_{t} \varepsilon_{t}^{\prime}\right)=I_{m}$. De esta manera la contribución de una variable j a la varianza del error de predicción de una variable i está determinada por:

$$
\frac{\psi_{0, i j}^{2}+\psi_{1, i j}^{2}+\cdots+\psi_{n-1, i j}^{2}}{\sum_{k=0}^{n-1} \psi_{k, i 1}^{2}+\psi_{k, i 2}^{2}+\cdots+\psi_{k, i m}^{2}}
$$

Donde $\Psi_{k, j j}$ representa el elemento en la posición ij de la matriz $\Psi_{k}$. El denominador de la anterior ecuación corresponde a la varianza del error de predicción de la i-ésima variable del VAR. En la figura 4 se muestra la estimación de la descomposición de varianza del consumo agregado. Se puede observar que, si se realiza un pronóstico del consumo, la varianza de su error estará explicada en aproximadamente $20 \%$ a causa de las innovaciones del ingreso previsible. Por lo cual las variaciones en el ingreso previsible generan cambios en el consumo agregado de los hogares. 
Figura 4. Descomposición de varianza del error de predicción de las ventas minoristas

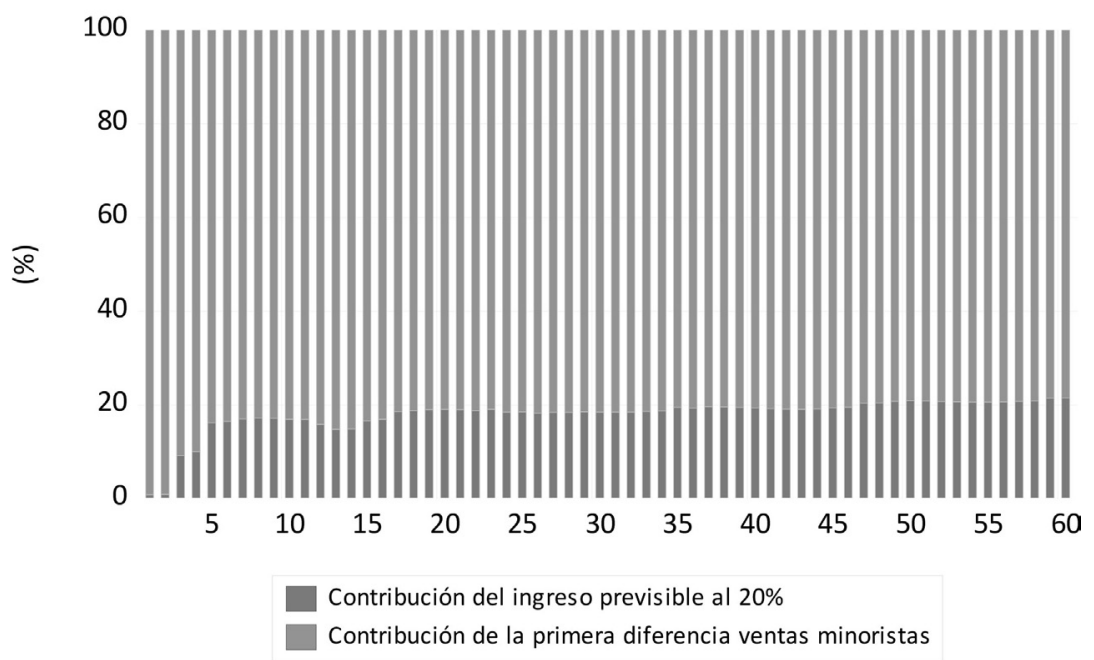

Meses del pronóstico

Fuente. Elaboración propia

La descomposición histórica de una variable endógena en un VAR puede realizarse con la representación $\operatorname{VMA}(\infty)$ con errores estructurales ortogonales (Burbidge et al., 1985). De acuerdo con dicha representación la jésima serie puede expresarse como:

$$
x_{j t}=\sum_{i=0}^{\infty} \psi_{i, j 1} e_{t-i, 1}+\psi_{i, j 2} e_{t-i, 2}+\cdots+\psi_{i, j m} e_{t-i, m}
$$

Por lo cual, la contribución del $k$-ésimo error estructural asociado a la $k$-ésima variable del VAR a $x_{j t}$ es igual a:

$$
\sum_{i=0}^{\infty} \psi_{i, j k} e_{t-i, k}
$$

Empíricamente dicha expresión se calcula usando la representación $\operatorname{VAR}(1)$ de un $\operatorname{VAR}(p)$, los estimadores de dichas matrices de coeficientes y los valores iniciales de las variables endógenas como base de comparación. La representación $\operatorname{VAR}(1)$ de un $\operatorname{VAR}(\mathrm{p})$ está dada por la siguiente expresión.

$$
r_{t}=A r_{t-1}+U_{t}
$$


Revista CIFE / ISSN: 0124-3551 e-ISSN: 2248-4914 / Bogotá-Colombia / Vol. 20 N.o 33 / julio-diciembre 2018 / pp. 17-37

Donde $Y_{t}$ es un vector de tamaño $m p x 1$ y $A$ es una matriz en bloques de tamaño $m p x m p$.

En la figura 5 se presenta la descomposición histórica del comercio minorista. La descomposición histórica puede interpretarse como lo valores que se habrían observado de una variable endógena si solo las innovaciones de una de ellas se hubiesen presentado. Se puede observar que el crecimiento del comercio causado por las innovaciones del ingreso previsible es relativamente constante y en promedio de $5.4 \%$ interanual. Por lo cual, los cambios en el ingreso previsible generan un incremento constante en el valor del consumo agregado. La estimación del ingreso previsible es un conjunto de variaciones periódicas en el ingreso y que se presentan al menos una vez en un año, generando un crecimiento histórico constante en el nivel de consumo.

Figura 5. Descomposición histórica, comercio minorista

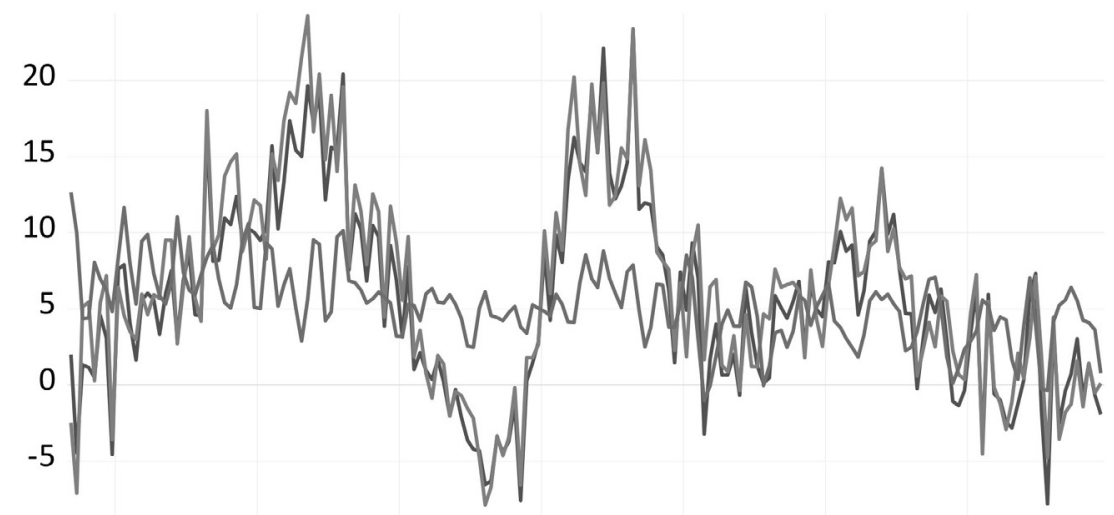

$\begin{array}{lllllllllllllll}03 & 04 & 05 & 06 & 07 & 08 & 09 & 10 & 11 & 12 & 13 & 14 & 15 & 16 & 17\end{array}$

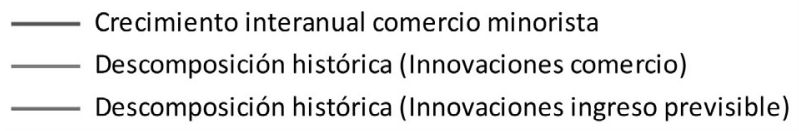

Fuente. Elaboración propia

Ahora bien, las funciones de impulso respuesta del $\operatorname{VAR}(\mathrm{p})$ puede estimarse utilizando sustitución recursiva con la representación $\operatorname{VAR}(1)$. De tal manera que:

$$
Y_{t}=A^{t} Y_{0}+\sum_{i=0}^{t} A^{i} U_{t-i}
$$

Si el sistema de ecuaciones es estable entonces $A^{t} \Upsilon_{0}=0$ y las respuestas tenderán hacia cero cuando $i \rightarrow \infty$. Los impulsos a las innovaciones se realizan en el vector $U_{t}$ en un momento en el tiempo. En la figura 6 se muestra la estimación de la respuesta de la 
diferencia del comercio minorista ante un impulso de una desviación estándar en el ingreso previsible. Las respuestas sombreadas son estadísticamente significativas al $95 \%$ de confianza. El efecto acumulado hasta de la respuesta de la diferencia del consumo es 0.21. Por ello incrementos en el ingreso previsible generan un aumento en el comercio minorista. La desviación del error es $\sqrt{0} 0.0029$. Así en el acumulado el comercio aumenta 4.2 puntos ante un incremento de 1 punto en el ingreso previsible.

Figura 6. Respuesta de la diferencia del comercio ante un impulso en el ingreso previsible

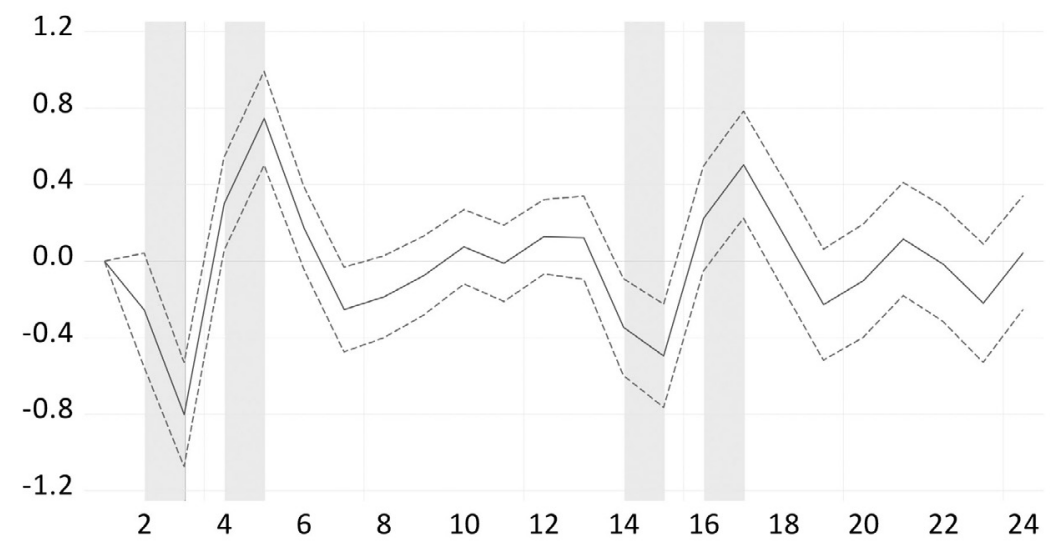

Fuente. Elaboración propia

\section{Conclusiones}

Utilizando estimaciones del ingreso previsible generadas por sus variaciones estacionales se encuentra evidencia a favor de la hipótesis de exceso de sensibilidad del consumo. Las variaciones previsibles del ingreso generadas por bonificaciones a los trabajadores, gastos en matrículas escolares, la agenda tributaria, contratos a término fijo, efectos calendario y costumbres sociales no se encuentran incorporadas en la senda de consumo de los agentes de la economía colombiana, a pesar de ser recurrentes cada año y totalmente previsibles. Esto puede explicarse a causa de la existencia de: i) ahorro precautelativo; ii) restricciones de liquidez; iii) alejamiento de la optimización de los consumidores y; iv) formación de hábitos de consumo. Se sugiere en futuras investigaciones identificar la importancia relativa de cada una de estas causas.

Dicha relación implica que los impuestos o subsidios generan un cambio en el consumo sin importar si son permanentes o transitorios, haciendo el consumo sin desestacionalizar más errático, debido a que los consumidores no incorporan este cambio previsible 
Revista CIFE / ISSN: 0124-3551 e-ISSN: 2248-4914 / Bogotá-Colombia / Vol. 20 N.o 33 / julio-diciembre 2018 / pp. 17-37

o imprevisible para suavizar su senda de consumo óptima. Las variaciones previsibles en el ingreso disponible generan variaciones adicionales en el consumo de los hogares.

\section{Referencias bibliográficas}

Agarwal, S., Liu, C. y Souleles, N. S. (2007). The reaction of consumer spending and debt to tax rebates-evidence from consumer credit data. Fournal of Political Economy, 115(6), 986-1019.

Attanasio, O. P. y Weber, G. (2010). Consumption and saving: models of intertemporal allocation and their implications for public policy. Fournal of Economic literature, 48(3), 693-751.

Browning, M. y Collado, M. D. (2001). The response of expenditures to anticipated income changes: panel data estimates. American Economic Review, 91(3), 681-692.

Campbell, J. Y. y Mankiw, N. G. (1989). Consumption, income, and interest rates: Reinterpreting the time series evidence. NBER macroeconomics annual, 4, 185-216.

Canzoneri, M. B., Cumby, R. E. y Diba, B. T. (2007). Euler equations and money market interest rates: A challenge for monetary policy models. Fournal of Monetary Economics, 54(7), 1863-1881.

Carroll, G. D. (1997). Buffer-stock saving and the life cycle/permanent income hypothesis. The Quarterly Journal of Economics, 112(1), 1-55.

Deaton, D. (1989). Saving and Liquidity Constraints. National Bureau of Economic Research Working. Paper n. ${ }^{\circ} 3196$.

Flavin, M. A. (1981). The adjustment of consumption to changing expectations about future income. Fournal of Political Economy, 89(5), 974-1009.

Friedman, M. (1957). The permanent income hypothesis. A theory of the consumption function (pp. 20-37). Princeton University Press, National Bureau of Economic Research.

Hall, R. E. (1978). Stochastic implications of the life cycle-permanent income hypothesis: Theory and evidence. Fournal of Political Economy, 86(6), 971-987.

Hsieh, C. T. (2003). Do consumers react to anticipated income changes? Evidence from the Alaska permanent fund. American Economic Review, 93(1), 397-405. 
Johnson, D. S., Parker, J. A. y Souleles, N. S. (2006). Household expenditure and the income tax rebates of 2001. American Economic Review, 96(5), 1589-1610.

Keynes, J. M. (1936). Teoría general de la ocupación, el interés y el dinero. Fondo de cultura económica.

Laibson, D. (1997). Golden eggs and hyperbolic discounting. The Quarterly fournal of Economics, $112(2), 443-478$.

Leland, H. E. (1978). Saving and uncertainty: The precautionary demand for saving. Uncertainty in Economics, 127, 129-139.

Lütkepohl, H. (2005). New introduction to multiple time series analysis. Springer Science y Business Media, 100-250.

Mankiw, N. G. (2014). Macroeconomía. In A. Bosch (Ed.), (pp. 327-425).

McCrorie, J. R. y Chambers, M. J. (2006). Granger causality and the sampling of economic processes. Journal of Econometrics, 132(2), 311-336.

Parker, J. A. (1999). The reaction of household consumption to predictable changes in social security taxes. American Economic Review, 89(4), 959-973.

Parker, J. A., Souleles, N. S., Johnson, D. S. y McClelland, R. (2013). Consumer spending and the economic stimulus payments of 2008. American Economic Review, 103(6), 25-53.

Paxson, C. H. (1992). Using weather variability to estimate the response of savings to transitory income in Thailand. The American Economic Review, 15-33.

Paxson, G. H. (1993). Consumption and income seasonality in Thailand. Fournal of Political Economy, 101(1), 39-72.

Priestley, M. B. (1981). Spectral analysis and time series. Academic Press. In Z. W. Birnbaum, E. Lukacs y G. Rebeco (Eds.), Macroeconomía: teoría y políticas (pp. 184-285). Pearson Educación.

Romer, D. (1996). Advanced macroeconomic theory. McGraw-Hill.

Shapiro, M. D. y Slemrod, J. (2003). Consumer response to tax rebates. American Economic Review, 93(1), 381-396.

Shea, J. (1995). Myopia, liquidity constraints, and aggregate consumption: a simple test. Journal of money, credit and banking, 27(3), 798-805. 


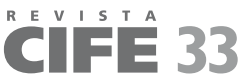

Revista CIFE / ISSN: 0124-3551 e-ISSN: 2248-4914 / Bogotá-Colombia / Vol. 20 N.o 33 / julio-diciembre 2018 / pp. 17-37

Skinner, J. (1988). Risky income, life cycle consumption, and precautionary savings. Journal of Monetary Economics, 22(2), 237-255.

Zeldes, S. P. (1989). Consumption and liquidity constraints: an empirical investigation. Journal of Political Economy, 97(2), 305-346.

\section{Anexo}

Estimación del VAR(15) con dicótomas estacionales y módulo del inverso de sus raíces.

\begin{tabular}{|c|c|c|}
\hline & (1) & (2) \\
\hline VARIABLES & previsible20 & dcomercio \\
\hline \multirow[t]{2}{*}{ L.previsible20 } & $2.237 * * *$ & $-4.695^{*}$ \\
\hline & $(0.0632)$ & $(2.720)$ \\
\hline \multirow[t]{2}{*}{ L2.previsible20 } & $-4.295^{* * * *}$ & -6.745 \\
\hline & $(0.138)$ & $(5.931)$ \\
\hline \multirow[t]{2}{*}{ L3.previsible20 } & $5.128 * * *$ & 14.56 \\
\hline & $(0.245)$ & $(10.56)$ \\
\hline \multirow[t]{2}{*}{ L4.previsible20 } & $-5.993^{* * * *}$ & $-29.25 * *$ \\
\hline & $(0.275)$ & (11.83) \\
\hline \multirow[t]{2}{*}{ L5.previsible20 } & $5.750 * * *$ & $27.71 * *$ \\
\hline & $(0.308)$ & $(13.26)$ \\
\hline \multirow[t]{2}{*}{ L6.previsible20 } & $-6.028 * * *$ & $-29.89 * *$ \\
\hline & $(0.280)$ & (12.05) \\
\hline \multirow{2}{*}{ L7.previsible20 } & 5.730 **** & $28.18 * *$ \\
\hline & $(0.309)$ & $(13.28)$ \\
\hline \multirow{2}{*}{ L8.previsible20 } & $-6.012 * * *$ & $-29.98 * *$ \\
\hline & $(0.278)$ & (11.96) \\
\hline \multirow{2}{*}{ L9.previsible20 } & $5.747 * * * *$ & $27.88^{* * *}$ \\
\hline & $(0.307)$ & $(13.22)$ \\
\hline \multirow[t]{2}{*}{ L10.previsible20 } & $-6.039 * * *$ & $-30.61 * *$ \\
\hline & $(0.280)$ & (12.07) \\
\hline
\end{tabular}




\begin{tabular}{|c|c|c|}
\hline & (1) & $(2)$ \\
\hline VARIABLES & previsible20 & dcomercio \\
\hline \multirow[t]{2}{*}{ L1 1.previsible20 } & $5.660 * * *$ & $28.34^{* * *}$ \\
\hline & $(0.309)$ & $(13.29)$ \\
\hline \multirow[t]{2}{*}{ L12.previsible20 } & $-4.895 * * *$ & $-30.37 * *$ \\
\hline & $(0.275)$ & $(11.82)$ \\
\hline \multirow[t]{2}{*}{ L13.previsible20 } & $3.301 * * *$ & $31.29 * * *$ \\
\hline & $(0.246)$ & $(10.60)$ \\
\hline \multirow[t]{2}{*}{ L14.previsible20 } & $-1.576^{* * * *}$ & $-21.86^{* * *}$ \\
\hline & $(0.137)$ & $(5.912)$ \\
\hline \multirow[t]{2}{*}{ L15.previsible20 } & $0.503^{* * *}$ & $12.00^{* * * *}$ \\
\hline & $(0.0653)$ & $(2.804)$ \\
\hline \multirow[t]{2}{*}{ L.dcomercio } & $-0.00677 * * *$ & $-0.539 * * *$ \\
\hline & $(0.00177)$ & $(0.0759)$ \\
\hline \multirow[t]{2}{*}{ L2.dcomercio } & $-0.00796^{* * *}$ & $-0.325^{* * * *}$ \\
\hline & $(0.00200)$ & $(0.0857)$ \\
\hline \multirow[t]{2}{*}{ L3.dcomercio } & 0.000144 & $-0.205^{* *}$ \\
\hline & $(0.00220)$ & $(0.0942)$ \\
\hline \multirow[t]{2}{*}{ L4.dcomercio } & $0.00668 * * * *$ & $-0.241 * * *$ \\
\hline & $(0.00212)$ & $(0.0909)$ \\
\hline \multirow[t]{2}{*}{ L5.dcomercio } & $0.00522 * *$ & -0.0750 \\
\hline & $(0.00215)$ & $(0.0925)$ \\
\hline \multirow[t]{2}{*}{ L6.dcomercio } & $0.00476 * *$ & $0.310^{* * *}$ \\
\hline & $(0.00215)$ & $(0.0920)$ \\
\hline \multirow[t]{2}{*}{ L7.dcomercio } & $0.0143^{* * *}$ & $0.201 * *$ \\
\hline & $(0.00194)$ & $(0.0835)$ \\
\hline \multirow[t]{2}{*}{ L8.dcomercio } & $0.0188 * * *$ & $0.170^{* *}$ \\
\hline & $(0.00174)$ & $(0.0750)$ \\
\hline \multirow[t]{2}{*}{ L9.dcomercio } & $0.00823^{* * * *}$ & 0.0646 \\
\hline & $(0.00220)$ & $(0.0946)$ \\
\hline
\end{tabular}


Revista CIFE / ISSN: 0124-3551 e-ISSN: 2248-4914 / Bogotá-Colombia / Vol. 20 N.o 33 / julio-diciembre 2018 / pp. 17-37

(1)

\section{VARIABLES}

L10.dcomercio

L11.dcomercio

L12.dcomercio

L13.dcomercio

L14.dcomercio

L15.dcomercio

\begin{tabular}{|c|c|c|}
\hline & $(1)$ & $(2)$ \\
\hline VARIABLES & previsible20 & dcomercio \\
\hline \multirow[t]{2}{*}{ L10.dcomercio } & $-0.00588 * * *$ & 0.141 \\
\hline & $(0.00219)$ & $(0.0952)$ \\
\hline \multirow[t]{2}{*}{ L1 1.dcomercio } & $-0.00746^{* * *}$ & $0.217^{* *}$ \\
\hline & $(0.00221)$ & $(0.0994)$ \\
\hline \multirow[t]{2}{*}{ L12.dcomercio } & $-0.00492^{* *}$ & $0.208 * *$ \\
\hline & $(0.00239)$ & $(0.102)$ \\
\hline \multirow[t]{2}{*}{ L13.dcomercio } & $-0.00888 * * *$ & -0.0609 \\
\hline & $(0.00234)$ & $(0.101)$ \\
\hline \multirow[t]{2}{*}{ L14.dcomercio } & $-0.0132^{* * *}$ & -0.0498 \\
\hline & $(0.00209)$ & $(0.0903)$ \\
\hline \multirow[t]{2}{*}{ L15.dcomercio } & $-0.00826^{* * *}$ & -0.0195 \\
\hline & (0.00188) & $(0.0805)$ \\
\hline \multirow[t]{2}{*}{$\mathrm{ml}$} & $-132.9 * * *$ & $-705.2^{* *}$ \\
\hline & $(6.632)$ & $(285.7)$ \\
\hline \multirow[t]{2}{*}{$\mathrm{m} 2$} & 0.213 & $-63.59 * * *$ \\
\hline & $(0.164)$ & (7.103) \\
\hline \multirow[t]{2}{*}{$\mathrm{m} 3$} & $-134.0 * * *$ & $-692.1 * *$ \\
\hline & $(6.696)$ & (288.4) \\
\hline \multirow[t]{2}{*}{$\mathrm{m} 4$} & -0.242 & $-37.74 * * *$ \\
\hline & $(0.158)$ & $(6.803)$ \\
\hline \multirow[t]{2}{*}{$\mathrm{m} 5$} & $-132.7 * * *$ & $-697.3^{* *}$ \\
\hline & $(6.632)$ & (285.7) \\
\hline \multirow[t]{2}{*}{$\mathrm{m} 6$} & $0.616^{* * *}$ & $-45.08 * * *$ \\
\hline & $(0.171)$ & $(7.419)$ \\
\hline \multirow[t]{2}{*}{$\mathrm{m} 7$} & $-133.2 * * *$ & $-676.4^{* *}$ \\
\hline & $(6.666)$ & $(287.1)$ \\
\hline \multirow[t]{2}{*}{ m8 } & $0.645^{* * *}$ & $-19.47 * * *$ \\
\hline & $(0.131)$ & $(5.658)$ \\
\hline
\end{tabular}

$$
\mathrm{m} 2
$$

\begin{tabular}{|c|c|c|}
\hline & $(1)$ & $(2)$ \\
\hline VARIABLES & previsible20 & dcomercio \\
\hline \multirow[t]{2}{*}{ L10.dcomercio } & $-0.00588 * * *$ & 0.141 \\
\hline & $(0.00219)$ & $(0.0952)$ \\
\hline \multirow[t]{2}{*}{ L1 1.dcomercio } & $-0.00746^{* * *}$ & $0.217^{* *}$ \\
\hline & $(0.00221)$ & $(0.0994)$ \\
\hline \multirow[t]{2}{*}{ L12.dcomercio } & $-0.00492^{* *}$ & $0.208 * *$ \\
\hline & $(0.00239)$ & $(0.102)$ \\
\hline \multirow[t]{2}{*}{ L13.dcomercio } & $-0.00888 * * *$ & -0.0609 \\
\hline & $(0.00234)$ & $(0.101)$ \\
\hline \multirow[t]{2}{*}{ L14.dcomercio } & $-0.0132^{* * *}$ & -0.0498 \\
\hline & $(0.00209)$ & $(0.0903)$ \\
\hline \multirow[t]{2}{*}{ L15.dcomercio } & $-0.00826^{* * *}$ & -0.0195 \\
\hline & (0.00188) & $(0.0805)$ \\
\hline \multirow[t]{2}{*}{$\mathrm{ml}$} & $-132.9 * * *$ & $-705.2^{* *}$ \\
\hline & $(6.632)$ & $(285.7)$ \\
\hline \multirow[t]{2}{*}{$\mathrm{m} 2$} & 0.213 & $-63.59 * * *$ \\
\hline & $(0.164)$ & (7.103) \\
\hline \multirow[t]{2}{*}{$\mathrm{m} 3$} & $-134.0 * * *$ & $-692.1 * *$ \\
\hline & $(6.696)$ & (288.4) \\
\hline \multirow[t]{2}{*}{$\mathrm{m} 4$} & -0.242 & $-37.74 * * *$ \\
\hline & $(0.158)$ & $(6.803)$ \\
\hline \multirow[t]{2}{*}{$\mathrm{m} 5$} & $-132.7 * * *$ & $-697.3^{* *}$ \\
\hline & $(6.632)$ & (285.7) \\
\hline \multirow[t]{2}{*}{$\mathrm{m} 6$} & $0.616^{* * *}$ & $-45.08 * * *$ \\
\hline & $(0.171)$ & $(7.419)$ \\
\hline \multirow[t]{2}{*}{$\mathrm{m} 7$} & $-133.2 * * *$ & $-676.4^{* *}$ \\
\hline & $(6.666)$ & $(287.1)$ \\
\hline \multirow[t]{2}{*}{ m8 } & $0.645^{* * *}$ & $-19.47 * * *$ \\
\hline & $(0.131)$ & $(5.658)$ \\
\hline
\end{tabular}

$\mathrm{m} 3$

\begin{tabular}{|c|c|c|}
\hline & $(1)$ & $(2)$ \\
\hline VARIABLES & previsible20 & dcomercio \\
\hline \multirow[t]{2}{*}{ L10.dcomercio } & $-0.00588 * * *$ & 0.141 \\
\hline & $(0.00219)$ & $(0.0952)$ \\
\hline \multirow[t]{2}{*}{ L1 1.dcomercio } & $-0.00746^{* * *}$ & $0.217^{* *}$ \\
\hline & $(0.00221)$ & $(0.0994)$ \\
\hline \multirow[t]{2}{*}{ L12.dcomercio } & $-0.00492^{* *}$ & $0.208 * *$ \\
\hline & $(0.00239)$ & $(0.102)$ \\
\hline \multirow[t]{2}{*}{ L13.dcomercio } & $-0.00888 * * *$ & -0.0609 \\
\hline & $(0.00234)$ & $(0.101)$ \\
\hline \multirow[t]{2}{*}{ L14.dcomercio } & $-0.0132^{* * *}$ & -0.0498 \\
\hline & $(0.00209)$ & $(0.0903)$ \\
\hline \multirow[t]{2}{*}{ L15.dcomercio } & $-0.00826^{* * *}$ & -0.0195 \\
\hline & (0.00188) & $(0.0805)$ \\
\hline \multirow[t]{2}{*}{$\mathrm{ml}$} & $-132.9 * * *$ & $-705.2^{* *}$ \\
\hline & $(6.632)$ & $(285.7)$ \\
\hline \multirow[t]{2}{*}{$\mathrm{m} 2$} & 0.213 & $-63.59 * * *$ \\
\hline & $(0.164)$ & (7.103) \\
\hline \multirow[t]{2}{*}{$\mathrm{m} 3$} & $-134.0 * * *$ & $-692.1 * *$ \\
\hline & $(6.696)$ & (288.4) \\
\hline \multirow[t]{2}{*}{$\mathrm{m} 4$} & -0.242 & $-37.74 * * *$ \\
\hline & $(0.158)$ & $(6.803)$ \\
\hline \multirow[t]{2}{*}{$\mathrm{m} 5$} & $-132.7 * * *$ & $-697.3^{* *}$ \\
\hline & $(6.632)$ & (285.7) \\
\hline \multirow[t]{2}{*}{$\mathrm{m} 6$} & $0.616^{* * *}$ & $-45.08 * * *$ \\
\hline & $(0.171)$ & $(7.419)$ \\
\hline \multirow[t]{2}{*}{$\mathrm{m} 7$} & $-133.2 * * *$ & $-676.4^{* *}$ \\
\hline & $(6.666)$ & $(287.1)$ \\
\hline \multirow[t]{2}{*}{ m8 } & $0.645^{* * *}$ & $-19.47 * * *$ \\
\hline & $(0.131)$ & $(5.658)$ \\
\hline
\end{tabular}

\begin{tabular}{|c|c|c|}
\hline & $(1)$ & $(2)$ \\
\hline VARIABLES & previsible20 & dcomercio \\
\hline \multirow[t]{2}{*}{ L10.dcomercio } & $-0.00588 * * *$ & 0.141 \\
\hline & $(0.00219)$ & $(0.0952)$ \\
\hline \multirow[t]{2}{*}{ L1 1.dcomercio } & $-0.00746^{* * *}$ & $0.217^{* *}$ \\
\hline & $(0.00221)$ & $(0.0994)$ \\
\hline \multirow[t]{2}{*}{ L12.dcomercio } & $-0.00492^{* *}$ & $0.208 * *$ \\
\hline & $(0.00239)$ & $(0.102)$ \\
\hline \multirow[t]{2}{*}{ L13.dcomercio } & $-0.00888 * * *$ & -0.0609 \\
\hline & $(0.00234)$ & $(0.101)$ \\
\hline \multirow[t]{2}{*}{ L14.dcomercio } & $-0.0132^{* * *}$ & -0.0498 \\
\hline & $(0.00209)$ & $(0.0903)$ \\
\hline \multirow[t]{2}{*}{ L15.dcomercio } & $-0.00826^{* * *}$ & -0.0195 \\
\hline & (0.00188) & $(0.0805)$ \\
\hline \multirow[t]{2}{*}{$\mathrm{ml}$} & $-132.9 * * *$ & $-705.2^{* *}$ \\
\hline & $(6.632)$ & $(285.7)$ \\
\hline \multirow[t]{2}{*}{$\mathrm{m} 2$} & 0.213 & $-63.59 * * *$ \\
\hline & $(0.164)$ & (7.103) \\
\hline \multirow[t]{2}{*}{$\mathrm{m} 3$} & $-134.0 * * *$ & $-692.1 * *$ \\
\hline & $(6.696)$ & (288.4) \\
\hline \multirow[t]{2}{*}{$\mathrm{m} 4$} & -0.242 & $-37.74 * * *$ \\
\hline & $(0.158)$ & $(6.803)$ \\
\hline \multirow[t]{2}{*}{$\mathrm{m} 5$} & $-132.7 * * *$ & $-697.3^{* *}$ \\
\hline & $(6.632)$ & (285.7) \\
\hline \multirow[t]{2}{*}{$\mathrm{m} 6$} & $0.616^{* * *}$ & $-45.08 * * *$ \\
\hline & $(0.171)$ & $(7.419)$ \\
\hline \multirow[t]{2}{*}{$\mathrm{m} 7$} & $-133.2 * * *$ & $-676.4^{* *}$ \\
\hline & $(6.666)$ & $(287.1)$ \\
\hline \multirow[t]{2}{*}{ m8 } & $0.645^{* * *}$ & $-19.47 * * *$ \\
\hline & $(0.131)$ & $(5.658)$ \\
\hline
\end{tabular}

\begin{tabular}{|c|c|c|}
\hline & $(1)$ & $(2)$ \\
\hline VARIABLES & previsible20 & dcomercio \\
\hline \multirow[t]{2}{*}{ L10.dcomercio } & $-0.00588 * * *$ & 0.141 \\
\hline & $(0.00219)$ & $(0.0952)$ \\
\hline \multirow[t]{2}{*}{ L1 1.dcomercio } & $-0.00746^{* * *}$ & $0.217^{* *}$ \\
\hline & $(0.00221)$ & $(0.0994)$ \\
\hline \multirow[t]{2}{*}{ L12.dcomercio } & $-0.00492^{* *}$ & $0.208 * *$ \\
\hline & $(0.00239)$ & $(0.102)$ \\
\hline \multirow[t]{2}{*}{ L13.dcomercio } & $-0.00888 * * *$ & -0.0609 \\
\hline & $(0.00234)$ & $(0.101)$ \\
\hline \multirow[t]{2}{*}{ L14.dcomercio } & $-0.0132^{* * *}$ & -0.0498 \\
\hline & $(0.00209)$ & $(0.0903)$ \\
\hline \multirow[t]{2}{*}{ L15.dcomercio } & $-0.00826^{* * *}$ & -0.0195 \\
\hline & (0.00188) & $(0.0805)$ \\
\hline \multirow[t]{2}{*}{$\mathrm{ml}$} & $-132.9 * * *$ & $-705.2^{* *}$ \\
\hline & $(6.632)$ & $(285.7)$ \\
\hline \multirow[t]{2}{*}{$\mathrm{m} 2$} & 0.213 & $-63.59 * * *$ \\
\hline & $(0.164)$ & (7.103) \\
\hline \multirow[t]{2}{*}{$\mathrm{m} 3$} & $-134.0 * * *$ & $-692.1 * *$ \\
\hline & $(6.696)$ & (288.4) \\
\hline \multirow[t]{2}{*}{$\mathrm{m} 4$} & -0.242 & $-37.74 * * *$ \\
\hline & $(0.158)$ & $(6.803)$ \\
\hline \multirow[t]{2}{*}{$\mathrm{m} 5$} & $-132.7 * * *$ & $-697.3^{* *}$ \\
\hline & $(6.632)$ & (285.7) \\
\hline \multirow[t]{2}{*}{$\mathrm{m} 6$} & $0.616^{* * *}$ & $-45.08 * * *$ \\
\hline & $(0.171)$ & $(7.419)$ \\
\hline \multirow[t]{2}{*}{$\mathrm{m} 7$} & $-133.2 * * *$ & $-676.4^{* *}$ \\
\hline & $(6.666)$ & $(287.1)$ \\
\hline \multirow[t]{2}{*}{ m8 } & $0.645^{* * *}$ & $-19.47 * * *$ \\
\hline & $(0.131)$ & $(5.658)$ \\
\hline
\end{tabular}

\begin{tabular}{|c|c|c|}
\hline & $(1)$ & $(2)$ \\
\hline VARIABLES & previsible20 & dcomercio \\
\hline \multirow[t]{2}{*}{ L10.dcomercio } & $-0.00588 * * *$ & 0.141 \\
\hline & $(0.00219)$ & $(0.0952)$ \\
\hline \multirow[t]{2}{*}{ L1 1.dcomercio } & $-0.00746^{* * *}$ & $0.217^{* *}$ \\
\hline & $(0.00221)$ & $(0.0994)$ \\
\hline \multirow[t]{2}{*}{ L12.dcomercio } & $-0.00492^{* *}$ & $0.208 * *$ \\
\hline & $(0.00239)$ & $(0.102)$ \\
\hline \multirow[t]{2}{*}{ L13.dcomercio } & $-0.00888 * * *$ & -0.0609 \\
\hline & $(0.00234)$ & $(0.101)$ \\
\hline \multirow[t]{2}{*}{ L14.dcomercio } & $-0.0132^{* * *}$ & -0.0498 \\
\hline & $(0.00209)$ & $(0.0903)$ \\
\hline \multirow[t]{2}{*}{ L15.dcomercio } & $-0.00826^{* * *}$ & -0.0195 \\
\hline & (0.00188) & $(0.0805)$ \\
\hline \multirow[t]{2}{*}{$\mathrm{ml}$} & $-132.9 * * *$ & $-705.2^{* *}$ \\
\hline & $(6.632)$ & $(285.7)$ \\
\hline \multirow[t]{2}{*}{$\mathrm{m} 2$} & 0.213 & $-63.59 * * *$ \\
\hline & $(0.164)$ & (7.103) \\
\hline \multirow[t]{2}{*}{$\mathrm{m} 3$} & $-134.0 * * *$ & $-692.1 * *$ \\
\hline & $(6.696)$ & (288.4) \\
\hline \multirow[t]{2}{*}{$\mathrm{m} 4$} & -0.242 & $-37.74 * * *$ \\
\hline & $(0.158)$ & $(6.803)$ \\
\hline \multirow[t]{2}{*}{$\mathrm{m} 5$} & $-132.7 * * *$ & $-697.3^{* *}$ \\
\hline & $(6.632)$ & (285.7) \\
\hline \multirow[t]{2}{*}{$\mathrm{m} 6$} & $0.616^{* * *}$ & $-45.08 * * *$ \\
\hline & $(0.171)$ & $(7.419)$ \\
\hline \multirow[t]{2}{*}{$\mathrm{m} 7$} & $-133.2 * * *$ & $-676.4^{* *}$ \\
\hline & $(6.666)$ & $(287.1)$ \\
\hline \multirow[t]{2}{*}{ m8 } & $0.645^{* * *}$ & $-19.47 * * *$ \\
\hline & $(0.131)$ & $(5.658)$ \\
\hline
\end{tabular}

\begin{tabular}{|c|c|c|}
\hline & $(1)$ & $(2)$ \\
\hline VARIABLES & previsible20 & dcomercio \\
\hline \multirow[t]{2}{*}{ L10.dcomercio } & $-0.00588 * * *$ & 0.141 \\
\hline & $(0.00219)$ & $(0.0952)$ \\
\hline \multirow[t]{2}{*}{ L1 1.dcomercio } & $-0.00746^{* * *}$ & $0.217^{* *}$ \\
\hline & $(0.00221)$ & $(0.0994)$ \\
\hline \multirow[t]{2}{*}{ L12.dcomercio } & $-0.00492^{* *}$ & $0.208 * *$ \\
\hline & $(0.00239)$ & $(0.102)$ \\
\hline \multirow[t]{2}{*}{ L13.dcomercio } & $-0.00888 * * *$ & -0.0609 \\
\hline & $(0.00234)$ & $(0.101)$ \\
\hline \multirow[t]{2}{*}{ L14.dcomercio } & $-0.0132^{* * *}$ & -0.0498 \\
\hline & $(0.00209)$ & $(0.0903)$ \\
\hline \multirow[t]{2}{*}{ L15.dcomercio } & $-0.00826^{* * *}$ & -0.0195 \\
\hline & (0.00188) & $(0.0805)$ \\
\hline \multirow[t]{2}{*}{$\mathrm{ml}$} & $-132.9 * * *$ & $-705.2^{* *}$ \\
\hline & $(6.632)$ & $(285.7)$ \\
\hline \multirow[t]{2}{*}{$\mathrm{m} 2$} & 0.213 & $-63.59 * * *$ \\
\hline & $(0.164)$ & (7.103) \\
\hline \multirow[t]{2}{*}{$\mathrm{m} 3$} & $-134.0 * * *$ & $-692.1 * *$ \\
\hline & $(6.696)$ & (288.4) \\
\hline \multirow[t]{2}{*}{$\mathrm{m} 4$} & -0.242 & $-37.74 * * *$ \\
\hline & $(0.158)$ & $(6.803)$ \\
\hline \multirow[t]{2}{*}{$\mathrm{m} 5$} & $-132.7 * * *$ & $-697.3^{* *}$ \\
\hline & $(6.632)$ & (285.7) \\
\hline \multirow[t]{2}{*}{$\mathrm{m} 6$} & $0.616^{* * *}$ & $-45.08 * * *$ \\
\hline & $(0.171)$ & $(7.419)$ \\
\hline \multirow[t]{2}{*}{$\mathrm{m} 7$} & $-133.2 * * *$ & $-676.4^{* *}$ \\
\hline & $(6.666)$ & $(287.1)$ \\
\hline \multirow[t]{2}{*}{ m8 } & $0.645^{* * *}$ & $-19.47 * * *$ \\
\hline & $(0.131)$ & $(5.658)$ \\
\hline
\end{tabular}

\begin{tabular}{|c|c|c|}
\hline & $(1)$ & $(2)$ \\
\hline VARIABLES & previsible20 & dcomercio \\
\hline \multirow[t]{2}{*}{ L10.dcomercio } & $-0.00588 * * *$ & 0.141 \\
\hline & $(0.00219)$ & $(0.0952)$ \\
\hline \multirow[t]{2}{*}{ L1 1.dcomercio } & $-0.00746^{* * *}$ & $0.217^{* *}$ \\
\hline & $(0.00221)$ & $(0.0994)$ \\
\hline \multirow[t]{2}{*}{ L12.dcomercio } & $-0.00492^{* *}$ & $0.208 * *$ \\
\hline & $(0.00239)$ & $(0.102)$ \\
\hline \multirow[t]{2}{*}{ L13.dcomercio } & $-0.00888 * * *$ & -0.0609 \\
\hline & $(0.00234)$ & $(0.101)$ \\
\hline \multirow[t]{2}{*}{ L14.dcomercio } & $-0.0132^{* * *}$ & -0.0498 \\
\hline & $(0.00209)$ & $(0.0903)$ \\
\hline \multirow[t]{2}{*}{ L15.dcomercio } & $-0.00826^{* * *}$ & -0.0195 \\
\hline & (0.00188) & $(0.0805)$ \\
\hline \multirow[t]{2}{*}{$\mathrm{ml}$} & $-132.9 * * *$ & $-705.2^{* *}$ \\
\hline & $(6.632)$ & $(285.7)$ \\
\hline \multirow[t]{2}{*}{$\mathrm{m} 2$} & 0.213 & $-63.59 * * *$ \\
\hline & $(0.164)$ & (7.103) \\
\hline \multirow[t]{2}{*}{$\mathrm{m} 3$} & $-134.0 * * *$ & $-692.1 * *$ \\
\hline & $(6.696)$ & (288.4) \\
\hline \multirow[t]{2}{*}{$\mathrm{m} 4$} & -0.242 & $-37.74 * * *$ \\
\hline & $(0.158)$ & $(6.803)$ \\
\hline \multirow[t]{2}{*}{$\mathrm{m} 5$} & $-132.7 * * *$ & $-697.3^{* *}$ \\
\hline & $(6.632)$ & (285.7) \\
\hline \multirow[t]{2}{*}{$\mathrm{m} 6$} & $0.616^{* * *}$ & $-45.08 * * *$ \\
\hline & $(0.171)$ & $(7.419)$ \\
\hline \multirow[t]{2}{*}{$\mathrm{m} 7$} & $-133.2 * * *$ & $-676.4^{* *}$ \\
\hline & $(6.666)$ & $(287.1)$ \\
\hline \multirow[t]{2}{*}{ m8 } & $0.645^{* * *}$ & $-19.47 * * *$ \\
\hline & $(0.131)$ & $(5.658)$ \\
\hline
\end{tabular}

\begin{tabular}{|c|c|c|}
\hline & $(1)$ & $(2)$ \\
\hline VARIABLES & previsible20 & dcomercio \\
\hline \multirow[t]{2}{*}{ L10.dcomercio } & $-0.00588 * * *$ & 0.141 \\
\hline & $(0.00219)$ & $(0.0952)$ \\
\hline \multirow[t]{2}{*}{ L1 1.dcomercio } & $-0.00746^{* * *}$ & $0.217^{* *}$ \\
\hline & $(0.00221)$ & $(0.0994)$ \\
\hline \multirow[t]{2}{*}{ L12.dcomercio } & $-0.00492^{* *}$ & $0.208 * *$ \\
\hline & $(0.00239)$ & $(0.102)$ \\
\hline \multirow[t]{2}{*}{ L13.dcomercio } & $-0.00888 * * *$ & -0.0609 \\
\hline & $(0.00234)$ & $(0.101)$ \\
\hline \multirow[t]{2}{*}{ L14.dcomercio } & $-0.0132^{* * *}$ & -0.0498 \\
\hline & $(0.00209)$ & $(0.0903)$ \\
\hline \multirow[t]{2}{*}{ L15.dcomercio } & $-0.00826^{* * *}$ & -0.0195 \\
\hline & (0.00188) & $(0.0805)$ \\
\hline \multirow[t]{2}{*}{$\mathrm{ml}$} & $-132.9 * * *$ & $-705.2^{* *}$ \\
\hline & $(6.632)$ & $(285.7)$ \\
\hline \multirow[t]{2}{*}{$\mathrm{m} 2$} & 0.213 & $-63.59 * * *$ \\
\hline & $(0.164)$ & (7.103) \\
\hline \multirow[t]{2}{*}{$\mathrm{m} 3$} & $-134.0 * * *$ & $-692.1 * *$ \\
\hline & $(6.696)$ & (288.4) \\
\hline \multirow[t]{2}{*}{$\mathrm{m} 4$} & -0.242 & $-37.74 * * *$ \\
\hline & $(0.158)$ & $(6.803)$ \\
\hline \multirow[t]{2}{*}{$\mathrm{m} 5$} & $-132.7 * * *$ & $-697.3^{* *}$ \\
\hline & $(6.632)$ & (285.7) \\
\hline \multirow[t]{2}{*}{$\mathrm{m} 6$} & $0.616^{* * *}$ & $-45.08 * * *$ \\
\hline & $(0.171)$ & $(7.419)$ \\
\hline \multirow[t]{2}{*}{$\mathrm{m} 7$} & $-133.2 * * *$ & $-676.4^{* *}$ \\
\hline & $(6.666)$ & $(287.1)$ \\
\hline \multirow[t]{2}{*}{ m8 } & $0.645^{* * *}$ & $-19.47 * * *$ \\
\hline & $(0.131)$ & $(5.658)$ \\
\hline
\end{tabular}




\begin{tabular}{|lcc|}
\hline VARIABLES & $(\mathbf{1})$ & $(2)$ \\
\hline m9 & previsible20 & dcomercio \\
\hline m10 & $-132.1^{* * * *}$ & $-688.8^{* *}$ \\
\hline m1 & $(6.638)$ & $(285.9)$ \\
\hline Constant & $1.013^{* * * *}$ & $-30.23^{* * *}$ \\
\hline Observations & $(0.137)$ & $(6.057)$ \\
\hline R-squared & $-133.2^{* * * *}$ & $-693.1^{* *}$ \\
\hline
\end{tabular}

Standard errors in parentheses

$* * * \mathrm{p}<0.01, * * \mathrm{p}<0.05, * \mathrm{p}<0.1$

Inverse Roots of AR Characteristic Polynomial

$$
1.5
$$

1.0

$0.5 \quad \cdot \quad$

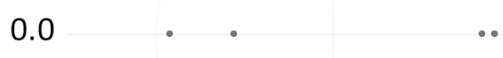

$-0.5 \quad \cdot \quad \cdot$

$-1.0$

$-1.5$

$\begin{array}{lll}-1 & 0 & 1\end{array}$

